\title{
A Classical Approach to the Modeling of Quantum Mass
}

\author{
Donald C. Chang \\ Div of LIFS, Hong Kong University of Science and Technology, Hong Kong, China \\ Email: bochang@ust.hk
}

Received September 1, 2013; revised September 29, 2013; accepted October 27, 2013

Copyright (C) 2013 Donald C. Chang. This is an open access article distributed under the Creative Commons Attribution License, which permits unrestricted use, distribution, and reproduction in any medium, provided the original work is properly cited.

\begin{abstract}
In modern physics, a particle is regarded as the quantum excitation of a field. Then, where does the mass of a particle come from? According to the Standard Model, a particle acquires mass through its interaction with the Higgs field. The rest mass of a free particle is essentially identified from the Klein-Gordon equation (through its associated Lagrangian density). Recently it was reported that a key feature of this theory (i.e., prediction of Higgs boson) is supported by experiments conducted at LHC. Nevertheless, there are still many questions about the Higgs model. In this paper, we would like to explore a different approach based on more classical concepts. We think mass should be treated on the same footing as momentum and energy, and the definition of mass should be strictly based on its association with the momentum. By postulating that all particles in nature (including fermions and bosons) are excitation waves of the vacuum medium, we propose a simple wave equation for a free particle. We find that the rest mass of the particle is associated with a "transverse wave number", and the Klein-Gordon equation can be derived from the general wave equation if one considers only the longitudinal component of the excitation wave. Implications of this model and its comparison with the Higgs model are discussed in this work.
\end{abstract}

Keywords: Mass; Vacuum; Quantum Excitation; Matter Wave; Particle-Wave Duality; Higgs Mechanism

\section{Introduction}

We know the universe is made up of particles; most of which have a rest mass (except for photons and possibly neutrinos). In the quantum field theory, a particle is regarded as an excitation wave of a field. Then, how can a particle acquire mass? According to the Standard Model (SM), the mass of the particle is acquired through their interaction with the Higgs field, the excitation of which is a scalar particle called "the Higgs boson" [1]. The Higgs particle has been theorized for a long time but was not verified in experiment until very recently. This is due to the very heavy mass of the Higgs particle and thus will require a very powerful accelerator to study it.

Experiments to hunt for the Higgs boson was finally started at CERN about three years ago using the newly built Large Hadron Collider (LHC). On July 2012, two experimental groups at LHC reported independently that they found a new particle with a mass of about $125 \mathrm{GeV}$, which could be the Higgs boson [2,3]. Earlier this year, their report was confirmed by further experimental data collected at LHC [4]. There was a lot of excitement in the physics community about their findings. Their results are considered to be a major triumph of the Standard
Model!

Yet, there are still many questions remaining to be answered. It is well aware in the physics community that the Standard Model (in the present form) has certain limitations. For example:

- It cannot explain gravitation.

- Some consider it to be ad hoc in nature, requiring 19 numerical constants whose values are arbitrary and can only be determined by experiments.

- The specifics of neutrino mass are still unclear. It is believed that explaining neutrino mass will require an additional 7 or 8 constants, which are also arbitrary parameters.

- The Standard Model cannot explain the huge amount of dark matter and dark energy observed in cosmology. It also has difficulty to explain the observed predominance of matter over anti-matter.

Thus, there is still a long way to go for physicists to develop a comprehensive theory that can explain our physical world from the sub-atomic scale to the cosmos scale. There is no wonder why many physicists in recent years are exploring new theories beyond the SM, (e.g., string theory, brane theory, super-symmetry, etc.). 
In this paper, we will explore a new approach to examine a specific problem in quantum physics. We will ask only one question, namely, what is the meaning of particle mass? We will first give a brief review on how particle acquires mass according to the Higgs model. We will explain why we need to consider alternative approaches. Then, we will present a new model which is based on more classical concepts. Finally, we will discuss the implications of this model.

\section{How Do Particles Acquire Mass in the Higgs Model?}

Let us first review briefly how mass is generated according to the Standard Model. Classically, mass is defined from its association with momentum. i.e.,

$$
\boldsymbol{p}=m \boldsymbol{v}
$$

This is not exactly how the quantum field theory works. In the Standard Model of particle physics, mass is a property associated with a specific field. The starting point is the energy-momentum relation attributed to the special theory of relativity (STR),

$$
E^{2}=p^{2} c^{2}+m^{2} c^{4}
$$

To construct the corresponding quantum wave equation, one can use the correspondence principle by converting the energy $(E)$ and momentum $(\boldsymbol{p})$ of a particle into operators of a field, such that $E \rightarrow i \hbar \partial / \partial t$ and $\boldsymbol{p} \rightarrow-i \hbar \nabla$, i.e., the wave equation is

$$
\left(\nabla^{2}-\frac{1}{c^{2}} \frac{\partial^{2}}{\partial t^{2}}\right) \phi-\left(\frac{m c}{\hbar}\right)^{2} \phi=0 .
$$

(Here $\hbar$ is Planck's constant divided by $2 \pi, \phi$ is the wave function of the field). This equation is called the Klein-Gordon equation. If we use a unit system such that $c=\hbar=1$, Equation (3) can be simply expressed as

$$
\left(\nabla^{2}-\frac{\partial^{2}}{\partial t^{2}}\right) \phi-m^{2} \phi=0 .
$$

In the SM, the concept of "field" is being generalized. Each type of particle is regarded as the excitation wave of its own field $\Phi$ (which can be a real or complex scalar function, a spinor, or a vector field). If one constructs a Lagrangian density of this field in the form of

$$
\mathcal{L}=\partial_{\mu} \Phi^{+} \partial^{\mu} \Phi-M^{2} \Phi^{+} \Phi
$$

(where $\mu$ is the usual summation index representing the 4-dimenional space), one can easily derive a generalized Klein-Gordon equation using Hamilton's principle (i.e., by applying the Euler-Lagrange equation),

$$
\left(\nabla^{2}-\frac{\partial^{2}}{\partial t^{2}}\right) \Phi-M^{2} \Phi=0 \text {. }
$$

Equation (6) is similar to Equation (4) if we interpret the parameter $M$ in Equation (6) as the "mass" of the excitation wave. In another word, the coefficient associated with the quadratic term $\Phi^{\dagger} \Phi$ in the Lagrangian density as shown in Equation (5) is identified as the square of the rest mass $\left(\mathrm{m}^{2}\right)$. Thus, in the quantum field theory, the rest mass of a free particle is essentially identified from the Klein-Gordon equation (through its associated Lagrangian density).

Using this as a guide line, one can investigate how a particle may acquire mass in the quantum field theory. Let us use the gauge boson as an example to demonstrate this mass-generating mechanism. In the SM, the gauge field is introduced solely to allow the Lagrangian density to be invariant under a local gauge transformation [5]. The excitations of this field are the gauge bosons. The gauge boson (such as photon) originally has no mass. The gauge boson acquires mass due to its interaction with a theoretical Higgs field, under the condition that the local symmetry of this field is broken. This mechanism was proposed by Higgs, and others in the mid 1960s [6-8].

The gauge field mediating the electro-weak interaction was introduced as a requirement of invariance of a Lagrangian density under local $S U(2)$ transformation as well as a local $U(1)$ transformation. This approach was first explored by Yang and Mills in 1954 [9], and was later developed into a theory to explain the unification of electro-weak interaction in leptons by Weinberg and Salem [10-11]. The following is a summary of essential steps used in the Higgs mechanism to allow gauge bosons to acquire mass:

Step 1: First, one may construct a general Lagrangian density which is Lorentz-invariant and can be renormalized,

$$
\mathcal{L}_{\Phi}=\partial_{\mu} \Phi^{+} \partial^{\mu} \Phi-V\left(\Phi^{+} \Phi\right)
$$

Here $\Phi$ is chosen to be a two-component field, (which is sometimes referred to as the Higgs field), i.e.

$$
\Phi=\left(\begin{array}{l}
\Phi_{A} \\
\Phi_{B}
\end{array}\right)
$$

where both $\Phi_{A}$ and $\Phi_{B}$ are complex scalar fields,

$$
\Phi_{A}=\phi_{1}+i \phi_{2}, \quad \Phi_{B}=\phi_{3}+i \phi_{4} .
$$

Step 2: If one requires this field to have a local $U(1)$ symmetry, one needs to introduce a vector gauge field $\boldsymbol{B}_{\mu}(x)$, which obeys certain transformation law. This procedure is analogous to the introduction of the vector potential $\boldsymbol{A}_{\mu}$ into electro-magnetism. If one makes a further requirement that this field is also invariant under a local $S U(2)$ transformation, one needs to introduce a second vector gauge field, $W_{\mu}(x)$. In order to make the Lagrangian density to become invariant under the $U(1) \times S U(2)$ 
transformation, the ordinary space-time derivative in Equation (7) will be replaced by the co-variant operator

$$
D_{\mu} \Phi=\left[\partial_{\mu}+\left(i g_{1} / 2\right) B_{\mu}+\left(i g_{2} / 2\right) W_{\mu}\right] \Phi,
$$

where $g_{1}$ and $g_{2}$ are coupling constants between the field $\Phi$ and the gauge fields associated with $U(1)$ symmetry and $S U(2)$ symmetry, respectively [12]. The locally gauge invariant Lagrangian density associated with the field $\Phi$ now becomes

$$
\mathcal{L}_{\Phi}=\left(D_{\mu} \Phi\right)^{+} D^{\mu} \Phi-V\left(\Phi^{+} \Phi\right)
$$

Step 3: Since the gauge fields are now entering the picture, one must also consider their contribution to the Lagrangian density. This dynamic contribution to the Lagrangian density associated with the gauge fields is shown to be [13]

$$
\mathcal{L}_{d y n}=-\frac{1}{4} B_{\mu v} B^{\mu v}-\frac{1}{8} \operatorname{Tr}\left(W_{\mu l} W^{\mu l}\right) .
$$

The total Lagrangian density for this system is then a sum of two contributions

$$
\mathcal{L}=\mathcal{L}_{\Phi}+\mathcal{L}_{\text {dyn }}
$$

Step 4: One may now consider what the potential energy term in the Lagrangian density should look like. In the Standard Model, $V$ is chosen to be in the following form (which is sometimes referred to as "the Higgs potential") [14],

$$
V\left(\Phi^{+} \Phi\right)=\frac{\lambda^{2}}{2 \phi_{0}^{2}}\left[\Phi^{+} \Phi-\phi_{0}^{2}\right]^{2},
$$

where $\phi_{o}$ is a fixed parameter specifying the ground state of the field, and $\lambda$ is another parameter associated with the coupling of the field to itself. One can now break the local $S U(2)$ symmetry by imposing certain restrictions on the wave function shown in Equation (7a), i.e., by requiring $\Phi_{A}=0$ and $\Phi_{B}$ is real [15]. The ground state is then

$$
\Phi_{\text {ground }}=\left(\begin{array}{c}
0 \\
\phi_{0}
\end{array}\right)
$$

and the excited state can be written as

$$
\Phi=\left(\begin{array}{c}
0 \\
\phi_{0}+h(x) / \sqrt{2}
\end{array}\right)
$$

where $h(x)$ is a real function. By combining Equations (9), (10) and (12) into Equation (11), and substituting Equation (14) into the combined equation, one can write down $\mathcal{L}$ in details in terms of the $h(x)$ field as well as the various components of the two vector gauge fields. By recombining the components of the $\boldsymbol{B}$ and $\boldsymbol{W}$ gauge fields into two new sets of vector fields $\boldsymbol{A}$ and $\boldsymbol{W} / \mathbf{Z}$, one can show that the Lagrangian density can be sorted out into two parts; $\mathcal{L}=\mathcal{L}_{1}+\mathcal{L}_{2}$, where $\mathcal{L}_{1}$ contains the Lagrangian density representing the free particles, while $\mathcal{L}_{2}$ contains all other parts (which are interpreted as interaction terms). $\mathcal{L}_{1}$ was shown to be [16]

$$
\mathcal{L}_{1}=\mathcal{L}_{\mathrm{H}}+\mathcal{L}_{\mathrm{Z}}+\mathcal{L}_{\mathrm{EM}}+\mathcal{L}_{\mathrm{W}}
$$

where

$$
\begin{gathered}
\mathcal{L}_{\mathrm{H}}=\frac{1}{2} \partial_{\mu} h \partial^{\mu} h-\lambda^{2} h^{2} \\
\mathcal{L}_{\mathrm{Z}}=-\frac{1}{4} Z_{\mu \nu} Z^{\mu v}+\frac{1}{4} \phi_{0}^{2}\left(g_{1}^{2}+g_{2}^{2}\right) Z_{\mu} Z^{\mu}, \\
\mathcal{L}_{\mathrm{EM}}=-\frac{1}{4} A_{\mu v} A^{\mu v} \\
\mathcal{L}_{W} \\
=-\frac{1}{2}\left[\left(D_{\mu} W_{v}^{+}\right) *-\left(D_{v} W_{\mu}^{+}\right) *\right]\left[\left(D^{\mu} W^{+v}\right)-\left(D^{v} W^{+\mu}\right)\right] . \\
+\frac{1}{2} g_{2}^{2} \phi_{0}^{2} W_{\mu}^{-} W^{\mu+}
\end{gathered}
$$

Here $A_{\mu}$ are components of the new vector gauge field A, while $Z_{\mu}$ and $W_{\mu}$ are components of the new vector gauge field $\boldsymbol{W} / \mathbf{Z}$; and we define $Z_{\mu \nu}=\partial_{\mu} Z_{v}-\partial_{\nu} Z_{\mu}$.

Step 5: Now one can identify the particles and their mass. Since each of these component Lagrangian densities (as shown in Equations (15a)-(15d)) looks like the $\mathcal{L}$ associated with a free particle as shown in Equation (5), one may interpret them as separated Lagrangian densities associated with different types of free particles. In the $\mathrm{SM}$, the excitations associated with the $h, Z_{\mu}, A_{\mu}$ and $W_{\mu}$ fields are identified as the Higgs boson, the $\mathrm{Z}$ boson, the photon, and the $\mathrm{W}^{+} / \mathrm{W}^{-}$boson, respectively. Also, by comparing Equations (15a)-(15d) with Equation (5), the mass of each particle associated with $\mathcal{L}_{\mathrm{H}}, \mathcal{L}_{\mathrm{Z}}, \mathcal{L}_{\mathrm{EM}}, \mathcal{L}_{\mathrm{W}}$ can be identified from the coefficient associated with their quadratic term of field strength. The results are summarized in Table 1.

Thus, according to the Standard Model, the gauge boson particles $\left(\mathrm{W}^{+}, \mathrm{W}^{-} \& \mathrm{Z}\right)$ acquire their mass solely due to their interaction with the Higgs field. Similar argument can be made on leptons [10,11] and quarks [17], although the theories involved are more complicated.

From the above review, one can easily see that, in the Higgs model, the rest mass of a particle is just a parameter associated with the strength of coupling between the particle field and the Higgs field. If this is true, we may have a philosophical problem. That is, the physical meaning of the rest mass $m$ would be intrinsically different from energy $E$ or momentum $p$. This does not seem to be very satisfactory in view of our traditional understanding of the physics concept. 
Table 1. Theoretical rest mass acquired by different particles under spontaneous breaking of local $U(1) \times S U(2)$ symmetry.

\begin{tabular}{ccccc}
\hline Lagrangian density & Field & Mediating force & Particle identification & Theoretical rest mass \\
\hline $\mathcal{L}_{H}$ & Massive scalar field & & Higgs boson & $M_{H}=\lambda \sqrt{2}$ \\
$\mathcal{L}_{Z}$ & Massive vector gauge field & Weak interaction & Gauge boson $Z$ & $M_{Z}=\frac{1}{\sqrt{2}} \phi_{0}\left(g_{1}^{2}+g_{2}^{2}\right)^{1 / 2}$ \\
$\mathcal{L}_{E M}$ & Massless vector gauge field & EM force & Photon & 0 \\
$\mathcal{L}_{W \pm}$ & Massive vector gauge field & Weak interaction & Gauge boson $W^{+/-}$ & $M_{W}=\frac{1}{\sqrt{2}} g_{2} \phi_{0}$ \\
\hline
\end{tabular}

\section{Why Do We Need to Consider an Alternative Approach?}

In this paper, we would like to explore a different approach to explain the origin of particle mass based on a less complicated physical picture. The motivation of doing this is mainly due to the following considerations: First, we think mass should be treated on the same footing as $E$ and $p$. Particularly, the rest mass $m$ should be associated with some sort of wave property, just like energy and momentum. Second, if each type of particle is regarded as the excitation of one distinct field, it would require a large number of fields in the universe. This is not very attractive in concept; and will require very convincing experimental justification. So far, we can only measure the physical variables of one field, namely, the electromagnetic field. All other fields are hypothetical and their properties are not based on experimental observation.

Third, it is not clear in the Higgs model what the property of the vacuum is. In the quantum field theory, the vacuum is regarded as the ground state of all fields. This in essence regards the vacuum as an invisible junkyard, which is used to accommodate all unexplainable parameters in the theory. This makes the vacuum very complicated. For example, the quantum field theory requires the vacuum to contain the zero-point energy of all particles. This means that, at each point of space-time, the vacuum should contain an infinite amount of energy. Does it make sense? In addition, the SM requires the vacuum to contain an infinite number of virtual particlepairs (with different $E$ and $p$ ) at any given point of spacetime. What is the physical basis of that?

Finally, there seems to be a conceptual difficulty in the Higgs model. It is very difficult to use common-sense ideas to explain to a layman how particle may acquire mass through the Higgs mechanism. In the last 40 years, various analogies have been invented to describe the particle's interaction with the Higgs field, including analogies with well known symmetry-breaking effects, such as the formation of rainbow from sunlight, separation of color using a prism, motion of a charge particle in an electric fields, and resistance affecting some objects moving through syrup or snow [18]. However, none of these analogies appears to be satisfactory. In fact, analogies based on simple resistance to motion are misleading, since the Higgs field does not work by resisting motion of the particle.

\section{What Is Our Philosophical Starting Point?}

We think a better approach to explain the particle mass would be a model that can take into consideration of the following specific points:

- Mass should be treated on the same footing as $E$ and p.

- The properties of the fields should be based on wellgrounded experimental observations rather than theoretical (or mathematical) convenience.

- The number of fields should be greatly limited.

- Properties of the vacuum should be more clearly defined.

- The theory should provide a physical mechanism for explaining how particles can be created or annihilated in the vacuum.

In order to satisfy the foregoing requirements, we would like to explore a new approach to explain the origin of particle mass. The starting point of this new model is to assume that:

1) The vacuum is a continuous medium, which can be excited by an energetic stimulation. The excitation wave can travel within this medium in long distance without energy loss (just like electrons in a superconductor or phonons in a superfluid). We may call this physical medium the " $V$-medium".

2) The excitation waves of the $V$-medium behave like particles in a macroscopic view. In another word, these particles are equivalent to solitons or quasi-particles that were already observed in many physical systems. This assumption is inspired by the fact that many particles are known to have a dual property of wave and corpuscle.

3) Different excitation waves of the $V$-medium make up different types of particles observed in the physical world. Both fermions and bosons are excitation waves of the same vacuum medium. This assumption is based on the fact that both photons and sub-atomic particles (like electrons and neutrons) behave as physical waves in diffraction experiments. Also, electron-positron pair and 
photon can be converted between each other.

4) The form of the general wave equation describing the vacuum is determined solely by the physical properties of the $V$-medium. Since solutions of this wave equation represent particles of different mass, this equation should not contain the parameter $m$ in it.

5) The mass $m$ is more like an eigen value; it should only emerge in the subsequent wave equation describing the motion of a particle with given mass. (This is analogues to the case that, in the classical quantum theory, the energy $E$ is an eigen value. $E$ only appears in the time-independent Schrödinger equation, $H \psi=E \psi$, but not in the more general time-dependent Schrödinger equation) [19].

Our assumption of a $V$-medium may remind people of the "ether" hypothesis proposed in the 19th century, which played a prominent role in the study of radiation before the development of quantum physics [20]. Almost two hundred years ago, many scientists had concluded that light was made up of oscillating waves. Then, there must be a medium that carries this wave of light. This invisible medium was called "ether". Some physicists, such as Young and Fresnel, suggested that the ether possesses the power of resisting attempt to distort its shape. In another word, the ether behaves like an elastic solid. Before the 20th century, many prominent physicists (and mathematicians), including Faraday, Helmholtz, Maxwell, Stokes, Cauchy, Poisson, Gauss and Riemann, had actively participated in studying various aspects of the ether theory [20].

The concept of ether, however, fell out of favor in the beginning of the 20th century. This was because it could not overcome two very serious challenges. First, it could not explain how large objects (like planets) can move through the ether. If ether is like an elastic solid, how can planets in their orbital motion travel through the ether at immense speed without resistance? Stokes suggested that ether may behave like shoemaker's wax; it was rigid enough to be capable of elastic vibration, but is yet sufficiently plastic to allow other slower bodies to pass it. The motion of a planet, of course, is much slower than light. This explanation, however, was rather superficial and was not convincing to most physicists.

Second, a more serious problem was that all attempts to detect the earth's movement through the ether failed. The existence of ether was examined in a famous experiment conducted by Michelson and Morely (1887) [21]. They developed a very sensitive instrument called "the Michelson interferometer" to measure the difference of light speeds in two pathways perpendicular to each other. When this interferometer moves through the hypothetical ether, one would expect that the light speed along the pathway parallel to the motion of ether should be different from the pathway perpendicular to it. The experimental results, however, was very surprising; there was no measurable difference between the light speeds of the two pathways, regardless how one may orient the interferometer to different directions related to the earth's orbit. The null results of their experiment were widely interpreted as a failure to observe the existence of ether [22].

If the ether theory was already discredited a long time ago, why would we propose the presence of a vacuum medium in this work? There is a major difference between our hypothesis and the ether theory. In the 19th century, the ether was hypothesized to carry the light wave in the vacuum between matters. The ether had nothing to do with matter itself. In our model, not only electromagnetic waves (photons), but all particles, including sub-atomic particles that make up matters in the universe, are excitation waves of the $V$-medium. Thus, when matters (such as a planet) move through the vacuum medium, there is no resistance. (This is analogous to sound waves travelling through a solid, electrons moving inside a superconductor, or phonons moving through a superfluid.)

Then, there is no wonder that any attempt to detect the earth's movement through the vacuum medium will fail. This explains the null results of experiments like those conducted by Michelson and Morely [21].

\section{Basic Theory of Our Model}

Based on the foregoing considerations, we can construct a new model to investigate the possible meaning of particle mass. As a formal starting point, we can say that our theory is based on two conceptual postulates [23,24]:

1) All particles found in nature (including fermions and bosons) are excitation waves of the vacuum medium (i.e., the $V$-medium).

2) Different types of particles (which make up either matter or radiation) are different excitation modes of the same vacuum medium.

The second postulate implies that the equation of motion for all particles should be identical and this equation is essentially determined by the physical properties of the vacuum medium. In the following, we will outline our theoretical approach step by step.

Step 1: Determining the wave equation of the vacuum medium

Our first job is to consider how to find the wave equation of the vacuum medium. What does this wave equation look like? This is a very difficult question, since we have very limited knowledge about the physical properties of the $V$-medium at this point. We can only make reasonable assumptions based on our understanding of other well known physical systems. As we mentioned before, the equation of motion of a given system can be generated based on Hamilton's principle by constructing 
a proper Lagrangian density, which is defined as

$$
\mathcal{L}=T-V \text {. }
$$

where $T$ is the kinetic energy and $V$ is the potential energy. Like in most physical systems, we may separate $\mathcal{L}$ into two parts

$$
\mathcal{L}=\mathcal{L}_{1}+\mathcal{L}_{2}
$$

where $\mathcal{L}_{1}$ is the Lagrangian density describing the motion of excitation waves representing free particles, while $\mathcal{L}_{2}$ represents the interaction terms. What should be the proper forms of $\mathcal{L}_{1}$ and $\mathcal{L}_{2}$ for the vacuum medium? We can make some educated guesses by considering several well-known facts and reasonable conjectures. First, the wave equation of the $V$-medium must be isotropic, since there is no preferred direction in the universe. Second, we already know the wave equation of at least one type of particle, namely, the photon. This can provide a very useful hint. Third, we know that the Klein-Gordon equation cannot be the general wave equation of the $V$-medium, since it contains the parameter $m$. But, we also know that the electron theory of Dirac is highly successful; and Dirac's theory is based on the Klein-Gordon equation [25]. So, the general wave equation of the vacuum must be capable to give the Klein-Gordon equation when the concerned wave function is reduced to describing only one specific type of particle (such as an electron).

Based on the above considerations, we can greatly narrow our choice for the wave equation of the $V$-medium. We speculate that $\mathcal{L}_{1}$ must be in the form

$$
\mathcal{L}_{1}=\partial_{\mu} \Phi^{+} \partial^{\mu} \Phi
$$

This is a very common Lagrangian density used to describe the elastic wave of an isotropic medium. Here, the kinetic energy is represented by the time derivative part, while the space derivative part represents the potential energy, which arises solely due to the stretching or compressing of the physical medium. By substituting $\mathcal{L}_{1}$ into the Euler-Lagrange equation, we can obtain the wave equation for a free particle, which is

$$
\square \psi=0,
$$

where $\square \equiv \nabla^{2}-\frac{1}{c^{2}} \frac{\partial^{2}}{\partial t^{2}}$ is the wave operator. Interestingly, this is exactly the wave equation of a photon, if $\psi$ is taken as a component of the vector potential $\left(A_{\mu}\right)$ of the electro-magnetic field.

What is the form of $\mathcal{L}_{2}$ in Equation (17)? This is not exactly known at this point, but we can have some rough ideas. First, it must be extremely short-ranged. In quantum physics, one considers mainly three forces: the EM force, the weak interaction and the strong interaction. Since we already shown that $\mathcal{L}_{1}$ can give entirely the ex- citation wave of the EM field, $\mathcal{L}_{2}$ is most likely to be relevant only for the weak and strong interactions. That means that it must be very short-ranged. Second, $\mathcal{L}_{2}$ is mainly responsible for interactions between different particles. We know that, in the absence of an external field, a free particle can travel for a long distance in vacuum at a straight line, without altering its energy and momentum. This motion is essentially described by the wave equation generated from $\mathcal{L}_{1}$ alone. So $\mathcal{L}_{2}$ is likely to be responsible for the interaction between particles. Based on the above considerations, one can treat $\mathcal{L}_{2}$ as a perturbation term, which is expected to be very small and can be ignored if we consider only the asymptotic behavior of a free particle.

Thus, under the condition that the system contains only one free particle (i.e., no particle-particle interaction) and we consider only the asymptotic behavior of the excitation wave (in spatial dimensions larger than an atomic nucleus), we can hypothesize that the wave equation of a free particle in the vacuum medium is essentially in the form of Equation (19). Here, the wave function $\psi$ is not limited to be a scalar function; it could represent different types of physical fields (which are different modes of stress of the $V$-medium). The mathematical representation of these fields could be a scalar, a vector, a pseudovector (like a spinor), or even a tensor.

In summary, one may justify our hypothesis based on several considerations. First, the proposed wave Equation is isotropic. Second, Equation (19) is valid for at least one type of particle, namely, the photon. Third, this equation can be derived directly from the Maxwell equations, which accurately describe the electro-magnetic properties of the vacuum based on experimental observations. (Here, the light velocity $c$ is determined directly from the dielectric constant and the permeability of the vacuum medium.) Forth, according to our second postulate, different particles are just different excitation modes of the same vacuum medium. If this is true, the motion of particles other than photon should also obey the same wave equation.

We may call Equation (19) as the 4-dimensional Laplace equation. It is probably the most general form of wave equation found in many isotropic physical systems. Besides the propagation of EM fields, the same equation can be used to describe sound wave propagation in an elastic solid; one can simply replace the light speed with the speed of sound (which depends on the mechanical properties of the medium). In the 19th century, many physicists and mathematicians had devoted great efforts to study the elastic properties of ether and came up with various models to explain the optical effects. Some of them also had derived similar equations as Equation (19). For example, MacCullagh developed a dynamic theory in 1848 by considering the rotational elasticity of the vac- 
uum medium [20]. He derived a wave equation which looks exactly like Equation (19).

\section{Step 2: Solutions of the proposed wave equation}

We can now solve the wave equation of the vacuum and examine the properties of the wave function. For reason of simplicity, hereafter we will treat the wave function in Equation (19) as a scalar function.

The simplest solution of Equation (19) is a plane wave

$$
\psi_{\boldsymbol{k}} \sim \mathrm{e}^{i(\boldsymbol{k} \cdot \boldsymbol{x}-\omega t)},
$$

where $\boldsymbol{k}$ and $\omega$ are the wave vector and frequency, respectively. This plane wave solution is commonly used to represent a photon. It, however, does not seem to be appropriate in describing a particle with nonzero rest mass, since such a particle would behave like a mass point in the classical limit. A massive particle must have a limited cross-section. Then, the wave representing the particle should not be uniform in the transverse plane. (That is, the probability of finding the particle is expected to be highest at its trajectory). This expectation suggests that the wave function of a free particle should depend not only on the spatial coordinate parallel to its trajectory (i.e., $\hat{\boldsymbol{k}} \cdot \boldsymbol{x}$ ), but also on the coordinates in the transverse plane $(\hat{\boldsymbol{k}} \times \boldsymbol{x})$.

Furthermore, since the trajectory of a free particle is a straight line, only one direction (i.e. the direction of motion, $\hat{\boldsymbol{k}}$ ) is specified. The wave function must have a cylindrical symmetry. Thus, one can assume that the general wave function representing a free particle must have the form

$$
\psi_{\hat{\boldsymbol{k}}}(\boldsymbol{x}, t)=\psi_{L}(\hat{\boldsymbol{k}} \cdot \boldsymbol{x}, t) \psi_{T}(\hat{\boldsymbol{k}} \times \boldsymbol{x}),
$$

where $\psi_{L}$ is the longitudinal component of the wave function which describes the travelling wave along the particle's trajectory, and $\psi_{T}$ is the transverse component of the wave function which determines the probability density of the particle at the transverse plane. Substituting Equation (21) into Equation (19), one has

$$
\begin{aligned}
& \nabla^{2}\left[\psi_{T}(\hat{\boldsymbol{k}} \times \boldsymbol{x}) \psi_{L}(\hat{\boldsymbol{k}} \cdot \boldsymbol{x}, t)\right] \\
& -\frac{\psi_{T}(\hat{\boldsymbol{k}} \times \boldsymbol{x})}{c^{2}} \frac{\partial^{2}}{\partial t^{2}} \psi_{L}(\hat{\boldsymbol{k}} \cdot \boldsymbol{x}, t)=0
\end{aligned}
$$

After expanding the $\nabla^{2}$ term (keeping in mind that $\nabla \psi_{T} \cdot \nabla \psi_{L}$ vanishes) and dividing the whole equation by $\psi_{T} \cdot \psi_{L}$, one can rearrange Equation (22) to obtain

$$
\begin{aligned}
& \frac{1}{\psi_{L}(\hat{\boldsymbol{k}} \cdot \boldsymbol{x}, t)}\left[\nabla^{2}-\frac{1}{c^{2}} \frac{\partial^{2}}{\partial t^{2}}\right] \psi_{L}(\hat{\boldsymbol{k}} \cdot \boldsymbol{x}, t) \\
& =\frac{-1}{\psi_{T}(\hat{\boldsymbol{k}} \times \boldsymbol{x})} \nabla^{2} \psi_{T}(\hat{\boldsymbol{k}} \times \boldsymbol{x})
\end{aligned}
$$

The left-hand side of this equation is a function only of $\hat{\boldsymbol{k}} \cdot \boldsymbol{x}$ and $t$, while the right-hand side of this equation is a function only of $\hat{\boldsymbol{k}} \times \boldsymbol{x}$. Equation (23) holds only if both sides equal a constant, which we can denote it as $\ell^{2}$. Then, Equation (23) can be broken down into two simultaneous equations

$$
\left\{\begin{array}{l}
{\left[\nabla^{2}-\frac{1}{c^{2}} \frac{\partial^{2}}{\partial t^{2}}\right] \psi_{L}(\hat{\boldsymbol{k}} \cdot \boldsymbol{x}, t)=\ell^{2} \psi_{L}(\hat{\boldsymbol{k}} \cdot \boldsymbol{x}, t)} \\
\nabla^{2} \psi_{T}(\hat{\boldsymbol{k}} \times \boldsymbol{x})=-\ell^{2} \psi_{T}(\hat{\boldsymbol{k}} \times \boldsymbol{x})
\end{array}\right.
$$

which can be solved separately for $\psi_{L}$ and $\psi_{T}$. The solution of Equation (25) is

$$
\psi_{T}(\hat{\boldsymbol{k}} \times \boldsymbol{x}) \propto J_{n}(\ell r) \mathrm{e}^{ \pm i n \theta},
$$

where $J_{n}$ is the Bessel function of the first kind, and $n$ is an integer or half integer; $r$ and $\theta$ represent the radial distance and the azimuthal angle of the space vector in the transverse plane. The solution of Equation (24) is a plane wave

$$
\psi_{L}(\hat{\boldsymbol{k}} \cdot \boldsymbol{x}, t) \propto \mathrm{e}^{i(\boldsymbol{k} \cdot \boldsymbol{x}-\omega t)}
$$

where $\boldsymbol{k}=k \hat{\boldsymbol{k}}$ is a vector parallel to $\hat{\boldsymbol{k}}$ and

$$
\omega=\left(k^{2}+\ell^{2}\right)^{1 / 2} c
$$

By combining Equations (21), (26), and (27), the wave function of the $V$-medium thus becomes

$$
\psi_{\hat{k}}(\boldsymbol{x}, t)=a J_{n}(\ell r) \mathrm{e}^{ \pm i n \theta} \mathrm{e}^{i(\boldsymbol{k} \cdot \boldsymbol{x}-\omega t)}
$$

(where $a$ is a normalizing constant). As expected, the wave function of a free particle behaves like a travelling wave moving along the direction of its trajectory. But because of an added phase factor $n \theta$, the particle wave actually propagates in a helical fashion. The wave function as a whole thus behaves like a vortex. Also, due to the presence of the Bessel function, $\psi_{\hat{\mathbf{k}}}$ varies in a diminishing oscillating manner in the directions perpendicular to the particle's trajectory.

Step 3: Finding the physical meaning of the wave parameters in the classical limit

The wave function of Equation (29) contains four parameters, $\omega, \boldsymbol{k}, \ell$ and $n$. What are their physical meanings? From the correspondence principle [26], the energy $(E)$ and momentum $(\boldsymbol{p})$ of a particle in the classical limit can be obtained from the expectation values of the $E \rightarrow i \hbar \partial / \partial t$ and $\boldsymbol{p} \rightarrow-i \hbar \nabla$ operators, i.e.,

$$
E=\int \psi * i \hbar \frac{\partial}{\partial t} \psi \mathrm{d}^{3} x
$$

and

$$
\boldsymbol{p}=\int \psi * \frac{\hbar}{i} \nabla \psi \mathrm{d}^{3} x .
$$

Substituting Equation (29) into Equation (30A), one 
can easily show that

$$
E=\hbar \omega,
$$

which, of course, is identical to the Planck's relation. Similarly, by substituting Equation (29) into Equation (30B), one can obtain

$$
\boldsymbol{p}=\hbar \boldsymbol{k},
$$

which is identical to the de Broglie relation. These results are very encouraging. But what is the physical meaning of $\ell$ in the classical limit? From Equation (28), we know $\ell$ is closely related to $\omega$ and $\boldsymbol{k}$. By combining Equations (28), (31), and (32), one can obtain

$$
E^{2}=c^{2}\left(p^{2}+\hbar^{2} \ell^{2}\right)
$$

It is well known in wave mechanics that the particle velocity $(v)$ is determined by the group velocity of the wave packet [27], that is,

$$
v=\frac{\partial \omega}{\partial k}=\frac{\partial E}{\partial p}
$$

Combining Equations (33) and (34), one can solve for $\boldsymbol{p}$ and obtain

$$
p=\left[\frac{\hbar \ell / c}{\left(1-v^{2} / c^{2}\right)^{1 / 2}}\right] v .
$$

In the classical limit, the momentum $(p)$ is equal to the mass $(M)$ times the velocity $(v)$. Hence, we can identify the quantity within the bracket on the right-hand side of Equation (35) as mass, that is,

$$
M=\frac{\hbar \ell / c}{\left(1-v^{2} / c^{2}\right)^{1 / 2}}
$$

At $v=0, M$ equals the rest mass, $m$. Equation (36) then implies that

$$
m=\frac{\hbar \ell}{c}
$$

This indicates that the parameter $\ell$ is associated with the rest mass of the particle! This result appears to make good sense, since when we substitute Equation (37) into Equation (33), we have

$$
E^{2}=p^{2} c^{2}+m^{2} c^{4}
$$

which agrees exactly with the energy-momentum relationship obtained from the classical treatment of STR [28]. Furthermore, by substituting Equation (35) into Equation (33), one can solve for $E$ and obtain

$$
E=\frac{\hbar \ell c}{\left(1-v^{2} / c^{2}\right)^{1 / 2}}
$$

By substituting Equation (37) into Equations (35), (36), and (39), one can obtain the other "relativistic relations", i.e.

$$
\begin{aligned}
& \boldsymbol{p}=\gamma m \boldsymbol{v}, \\
& M=\gamma m
\end{aligned}
$$

and

$$
E=\gamma m c^{2},
$$

where $\gamma=\left(1-v^{2} / c^{2}\right)^{-1 / 2}$. By combining Equations (41) and (42), we have

$$
E=M c^{2}
$$

which is the well-known "Einstein's relation" of mass and energy.

Hence, from Equations (29) and (37), one can see that the rest mass of a particle is associated with the oscillation periodicity of the wave function in the transverse plane (which is perpendicular to the direction of movement of the particle). In short, among the four parameters found in the wave function as shown in Equation (29), we can identify each of them to a physical property of the particle in the classical limit. Namely, the wave vector $\boldsymbol{k}$ is associated with momentum $\boldsymbol{p}$, the frequency $\omega$ is associated with energy $E$, and the transverse wave number $\ell$ is associated with the rest mass $m$. Finally, one may ask what could be the physical meaning of the parameter $n$. It appears that $n$ is likely to be associated with the helicity of the free particle. Since $n$ is a quantum number conjugate to the angular coordinate $\theta$, dimensional analysis suggests that $n$ is associated with some sort of angular momentum. The helicity operator can be regarded as equivalent to an angular momentum operator about an axis of the particle's trajectory (in direction $\hat{\boldsymbol{k}}$ ). In our case the eigen value of this operator is $n \hbar$. Because of the phase factor $n \theta$, Equation (29) suggests that the excitation wave representing a free particle generally propagates in a helical fashion.

\section{Step 4: Interpretation of the Klein-Gordon equation}

From the above analysis, it is strongly suggested that the rest mass of a particle is associated with the periodic oscillation of the wave function in the transverse plane. This result appears to make good sense. First, such identification can directly lead to the well-known relativistic relations which are well supported by experiments. Second, one can easily see that mass is associated with a wave property of the excitation wave. This is very pleasing in consideration of the wave-particle duality. Third, from Equation (29), one can see that $m$ is associated with the inverse of an oscillation wavelength, since the Bessel function approximates a cosine function in the asymptotic limit, i.e.,

$$
J_{n}(\ell r) \rightarrow \cos (\ell r) /(\ell r)^{1 / 2}
$$

Thus, $m$ is associated with the curvature of the field 
(distortion in the $V$-medium) in the transverse plane caused by the excitation wave. By comparing Equation (37) with Equations (31) and (32), one can easily see that the physical meaning of $m$ is very similar to that of energy and momentum, since all of them are related to the curvature of the field. Thus, our result strongly suggests that the mass $m$ can indeed be treated on the same theoretical basis as $E$ and $p$.

After demonstrating that $\ell$ is connected with $m$, we can now identify the origin of the Klein-Gordon equation. By substituting Equation (37) into Equation (24), one can obtain

$$
\square \psi_{L}-\left(\frac{m c}{\hbar}\right)^{2} \psi_{L}=0,
$$

which is identical to the "Klein-Gordon equation" shown in Equation (3). One should notice that, by comparing Equations (3) and (44), the wave function described by the Klein-Gordon equation is just the longitudinal component of the travelling wave; it does not represent the entire wave function of a free particle.

In conclusion, the results of our analysis have several implications. First, by successfully deriving the KleinGordon equation, we can reconcile our model with that of the quantum field theory, even though our starting point is more classical. Second, the fact that we can reproduce the Planck's relation and the de Broglie relation suggest that our proposed wave equation for the vacuum medium is very reasonable. In another word, the free Lagrangian density constructed by us (i.e., Equation (18)) must be a good choice. Third, by following the derivation of Equation (44), it is clear that the Klein-Gordon equation is not a general wave equation of the vacuum medium; it can only describe the motion of a particle with a specific mass (i.e., when the parameter $\ell$ is already chosen). Forth, according to Equation (44), the wave function described by the Klein-Gordon equation, $\psi_{L}$, only represents the longitudinal part of the excitation wave. This part of the wave function contains mainly the positional information of the particle along its pathway; it does not describe the actual physical wave of the vacuum medium. Thus, the Klein-Gordon equation is mainly useful for describing the trajectory of the particle.

\section{Discussions}

The starting point of this work is that particles are regarded as quantum excitation waves of the vacuum medium (i.e., the $V$-medium). Thus, the equation of motion for all particles is determined by the physical properties of the vacuum. Based on a few general considerations, we proposed a wave equation for the $V$-medium under a simplified condition, i.e., when the vacuum contains only a single free particle. Solution of this wave equation suggests that, free particles with non-zero rest mass gen- erally travel through space in a straight line in a helical fashion. Only massless particle (such as photon) travels as a plane wave.

The physical meaning of particle mass is explored in this work. Our definition of mass is based on its association with momentum, just like what we learned in classical mechanics. We found that the rest mass of a particle is associated with a transverse wave number $\ell$, which is related to the periodic oscillation of the excitation wave in a plane perpendicular to the movement of the particle. Our finding appears to make good sense. It satisfies our requirement that the mass $m$ should be treated on the same footing as $E$ and $p$. It is well known that mass $m$ is a particle property; our model is able to naturally connect this particle property with a wave property of the excitation wave. This is highly satisfactory and consistent with the fact that all particles have the property of particlewave duality.

Our results can also provide a useful basis for explaining the origin of the Klein-Gordon equation, which comes out naturally from our model. We show that it is a simplified wave equation of the vacuum for a particle of specific $m$. This equation describes only the longitudinal component of the excitation wave. Once the KleinGordon equation is derived, one can then easily obtain the Dirac equation to describe the motion of electron [29]. Furthermore, based on the Dirac equation, the Schrödinger equation can be derived under the condition when the Coulomb potential energy and kinetic energy are small in comparison to the rest mass of the particle. This procedure had been shown elsewhere [30].

Finally, the well-known relativistic relations between energy, momentum and mass also emerge naturally in this model. But, we must point out that we did not use STR as the start point. Our proposed wave equation for the $V$-medium is based on the Maxwell equations (and the wave equation of an elastic solid). We obtain the relativistic relations because the wave equation chosen by us happens to be Lorentz invariant. Since the wave equation of a free particle comes from the Lagrangian density $\mathcal{L}_{1}$ alone, our choice only requires $\mathcal{L}_{1}$ to be Lorentz invariant. At this point we know very little about $\mathcal{L}_{2}$, the Lagrangian density responsible for particle interactions. There is no guarantee that the full Lagrangian density of the vacuum medium as shown in Equation (17) is Lorentz invariant. So, our requirement at this point is less stringent than the STR.

In summary, this work suggests that the vacuum is not an empty space with nothing in it. Instead, it is made of a physical medium that can propagate different sorts of elastic waves. This medium may appear to be invisible, because visibility requires photons, which appear only when the medium is in the excited state. We hypothesize that different types of particles observed in nature are just 
different modes of quantum excitations of this medium. At this point, the physical properties of the vacuum medium are not exactly known. It could behave like an elastic solid or a superfluid. How to find the detailed properties of the vacuum medium will be a major challenge in the future.

\section{Acknowledgements}

I am grateful to Profs. John Wheeler and H. E. Rorschach for their encouragement during the early stage of this work. I thank Profs. Zhaoqing Zhang and Xiangrong Wang for their comments. I also thank Mr. Wenbin Cao for his assistance. A preliminary version of this work was presented in the Joint APS/AAPT Meeting (1984) in USA [31] and in the 8th International CASYS Conference (2007) held in Liege, Belgium.

\section{REFERENCES}

[1] G. Z. Liu, G. Cheng, Physical Review B, Vol. 65, 2002, p. 13.

[2] ATLAS Collaboration, Physical Review B, Vol. 716, 2012, p. 1.

[3] CMS Collaboration, Physical Review B, Vol. 716, 2012, p. 30.

[4] ATLAS Collaboration, Physics Letters B, 2013. arXiv:1307.1427 [hep-ex]

[5] R. Oerter, "The Theory of Almost Everything: The Standard Model, the Unsung Triumph of Modern Physics," Penguin Group, 2006.

[6] F. Englert and R. Brout, Physical Review Letters, Vol. 13, 1964, pp. 321-323.

http://dx.doi.org/10.1103/PhysRevLett.13.321

[7] P. W. Higgs, Physical Review Letters, Vol. 13, 1964, pp. 508-509. http://dx.doi.org/10.1103/PhysRevLett.13.508

[8] G. S. Guralnik, C. R. Hagen and T. W. B. Kibble, Physical Review Letters, Vol. 13, 1964, pp. 585-587. http://dx.doi.org/10.1103/PhysRevLett.13.585

[9] C. N. Yang and R. Mills, Physical Review, Vol. 96, 1954, pp. 191-195. http://dx.doi.org/10.1103/PhysRev.96.191

[10] S. Weinberg, Physical Review Letters, Vol. 19, 1967, pp. 1264-1266. http://dx.doi.org/10.1103/PhysRevLett.19.1264

[11] A. Salam, "Elementary Particle Physics: Relativistic Groups and Analyticity," In: N. Svartholm, Ed., Eighth Nobel Symposium, Almquvist and Wiksell, Stockholm, 1968.

[12] W. N. Cottingham and D. A. Greenwood, "An Introduction to the Standard Model of Particle Physics," Cambridge University Press, Cambridge, 1998, pp. 103-105.
[13] W. N. Cottingham and D. A. Greenwood, "An Introduction to the Standard Model of Particle Physics," Cambridge University Press, Cambridge, 1998, pp. 105-106.

[14] J. Goldstone, "Field Theories with 'Superconductor' Solutions," Il Nuovo Cimento, Vol. 19, 1961, pp. 154-164. http://dx.doi.org/10.1007/BF02812722

[15] G. S. Guralnik, International Journal of Modern Physics $A$, Vol. 24, 2009, pp. 2601-2627. http://dx.doi.org/10.1142/S0217751X09045431

[16] W. N. Cottingham and D. A. Greenwood, "An Introduction to the Standard Model of Particle Physics," Cambridge University Press, Cambridge, 1998, pp. 107-109.

[17] W. N. Cottingham and D. A. Greenwood, "An Introduction to the Standard Model of Particle Physics," Cambridge University Press, Cambridge, 1998, pp. 131-139.

[18] J. Ellis, "What Is the Higgs Boson?" http://lybio.net/tag/john-ellis-what-is-the-higgs-boson-qu otes/

[19] A. Messiah, "Quantum Mechanics," John Wiley \& Sons, New York, 1965, pp. 59-72.

[20] E. Whittaker, "A History of the Theories of Aether and Electricity," Thomas Nelson and Sons Ltd., London, 1951.

[21] A. A. Michelson and E. W. Morley, "On the Relative Motion of the Earth and the Luminiferous Ether," American Journal of Science, Vol. 34, 1887, pp. 333-345. http://dx.doi.org/10.2475/ajs.s3-34.203.333

[22] A. P. French, "Special Relativity," Nelsen, London, 1968.

[23] D. C. Chang, "What Is Rest Mass in the Wave-Particle Duality? A Proposed Model," 2004.

ArXiv: physics/0404044

[24] D. C. Chang, "On the Wave Nature of Matter," 2005. ArXiv: physics/0505010

[25] P. A. M. Dirac, "The Principles of Quantum Mechanics," 4th Edition, Oxford University Press, Oxford, 1981.

[26] A. Messiah, "Quantum Mechanics," John Wiley \& Sons, New York, 1965, pp. 56-59.

[27] S. Nettel, "Wave Physics," 3rd Edition, Springer, Berlin, 2003, pp. 221-223. http://dx.doi.org/10.1007/978-3-662-05317-1

[28] A. Einstein, "Relativity. The Special and the General Theory," Three River Press, New York, 1961, pp. 1-64.

[29] J. J. Sakurai, “Advanced Quantum Mechanics," AddisonWesley, Reading, 1973, pp. 78-89.

[30] W. N. Cottingham and D. A. Greenwood, "An Introduction to the Standard Model of Particle Physics," Cambridge University Press, Cambridge, 1998, p. 72.

[31] D. C. Chang, Bulletin of the American Physical Society, Vol. 29, 1984, p. 6. 